\title{
Lexical access for low- and high-frequency words in Hebrew
}

\author{
ASHER KORIAT \\ University of Haifa, Haifa, Israel
}

\begin{abstract}
The hypothesis that phonological mediation is involved to a greater extent in the recognition of low- than in the recognition of high-frequency words was examined using Hebrew. Hebrew has two forms of spelling, pointed and unpointed, which differ greatly in the extent of phonological ambiguity, with the unpointed spelling lacking almost all vowel information. A lexical decision task was employed using target words that had only one pronunciation whether pointed or unpointed. Targets were either pointed or unpointed and were preceded by a prime, which for word targets, was either semantically related or unrelated. The results indicated the following: First, the advantage of pointed over unpointed spelling was larger for low-frequency than for high-frequency words, suggesting a stronger phonological mediation for low-frequency words. Second, the size of the pointing effect was independent of word length, suggesting that phonology is obtained on the basis of the printed word as a whole, by looking it up in a phonological lexicon. Third, response latency to nonwords was not affected by the presence or absence of pointing, suggesting that failure to locate the entry corresponding to a letter string in a phonological lexicon results in a NO decision. Fourth, presence of a related prime was not found to compensate for absence of pointing, suggesting that the activation of a word's representation in the semantic lexicon does not aid access to its corresponding entry in the phonological lexicon.
\end{abstract}

One of the most debated issues in research on reading concerns the extent to which phonological encoding is involved in the comprehension of printed words. The phonological recoding hypothesis (e.g., Rubenstien, Lewis, \& Rubenstein, 1971) posits that a printed word must be first coded into a phonological representation before lexical access can be achieved. In contrast, the direct, visual acces hypothesis assumes that meaning is derived directly from the graphemic pattern of a printed word (e.g., Baron, 1973). The third view combines these two, claiming that both modes of access are available to a reader, and that both are used in parallel, with lexical access being achieved by whichever process ends first. (Meyer, Schvaneveldt, \& Ruddy, 1974). This dual-access model is probably the most accepted today.

The general acceptance of the dual-access hypothesis has shifted interest from the question of which of the two modes of access is used to the question of when each of them determines lexical access. McCusker, Hillinger, and Bias (1981) reviewed several factors affecting the choice of mediating representation in reading. These include characteristics of the reading material, characteristics of the reader, and task demands. The present study focuses on one characteristic of the reading material, namely, word frequency. It has been proposed that difficult reading material is more likely to require phonological medi-

I am indebted to Avraham Papo for his technical assistance and to Joel Norman for his comments on an earlier draft. Requests for reprints should be sent to Asher Koriat, Department of Psychology, University of Haifa, Haifa, Israel.

-Steven W. Keele served as Action Editor for this manuscript. ation than simple material (Bradshaw, 1975). Thus, Hardyck and Petrinovich (1970) reported data that suggest, first, that subvocal speech is involved to a greater extent in the reading of difficult passages than in the reading of easy passages and, second, that the suppression of subvocal speech impairs comprehension of difficult passages more than that of easy passages. It is not clear, however, whether the increased use of subvocal speech with difficult material reflects a greater reliance on the phonological route to the lexicon or a greater postaccess use of the subvocal speech as a means to aid comprehension (cf. Kleiman, 1975; McCusker et al. 1981).

One simple index of difficulty of material is word frequency. It has been proposed (McCusker et al., 1981) that high-frequency words are normally accessed via a visual representation, with little or no phonological mediation, whereas low-frequency words require phonological recoding prior to lexical access. The most direct support for this proposition comes from McCusker, Holley-Wilcox, and Hillinger (1979). They argued that the advantage of high-frequency over low-frequency words in word recognition (the "word-frequency effect") is due, at least in part, to the fact that high-frequency words may be accessed rapidly using a graphemically based code, whereas low-frequency words have to rely on a slower, phonologically mediated process. Consistent with this proposition, they found that the size of the word-frequency effect was significantly smaller for an auditory lexical decision task ( $51 \mathrm{msec}$ ) than for a visual lexical decision task $(81 \mathrm{msec})$. This finding was taken to suggest that when visually based access is precluded, part of the ad- 
vantage of low-frequency over high-frequency words disappears.

Using Hebrew, the present study examines the interaction between word frequency and mode of lexical access. Hebrew orthography has one peculiar feature that makes it particularly useful in testing this hypothesis (cf. Koriat, 1984; Navon \& Shimron, 1981). It has two forms of spelling, pointed and unpointed. These differ greatly in the extent to which vowel information is spelled out. In the unpointed form, the letters convey mostly consonantal information, with vowel information being largely implicit. There are a few letters (e.g., Yod and Vav) that in some contexts serve in the same function as that of vowel letters in English, but they may in other contexts stand for consonants. Thus, with the unpointed spelling, much of the information necessary for a presemantic derivation of phonology is not expressed, resulting in a great number of homographs.

Pointed spelling is achieved by adding certain signs (referred to as pointing) to the unpointed spelling. These are placed below, above, or inside the letters. Pointing supplies mostly vowel information, but may also contain some consonantal information (e.g., the information necessary to distinguish $/ \mathrm{p} /$ and $/ \mathrm{f} /$ ). Although unpointed spelling is extremely ambiguous as far as phonological coding is concerned, pointed spelling may be classified as a very shallow orthography.

Both forms of Hebrew spelling are normally in use. Children's reading materials are usually pointed, and children begin by learning to read pointed spelling. Adult reading materials are typically unpointed, although sometimes (e.g., poetry, prayer books) the pointed spelling is conventionally used.

From a methodological point of view, the advantage of Hebrew is that we can manipulate the amount of phonological information available to a reader by using either the pointed or the unpointed forms of the exact same words.

In a previous study (Koriat, 1984), I examined the extent to which the presence of pointing aids lexical access. The idea was that if phonological recoding is a necessary precursor to lexical access, pointing, which reduces much of the phonological ambiguity, should prove beneficial. A lexical decision task was employed using words that had only one pronunciation whether pointed or not. Pointing, used as a between-subjects factor, was found to have little effect on response latency. This and other findings were interpreted as indicating that Hebrew word recognition is by and large direct, rather than involving phonological mediation. Pointing, however, had a significant effect on pronunciation latency, suggesting that it may aid in the presemantic derivation of phonology.

The possibility remains, however, that evidence for phonological mediation might be found for words whose frequencies were lower than the frequencies of words sampled in the previous study. In that study, the words employed were selected from the most frequently used 3,000 words in primary school material. Ther present study included, in addition, a set of low-frequency words. Also, in contrast to the previous study, in which pointing figured as a between-subjects variable, in the present study pointed and unpointed letter strings were mixed in the same list to preclude the adoption of different strategies for pointed and unpointed words.

The first aim of the present study was to examine the idea that if lexical access is visual for high-frequency words and phonological for low-frequency words, then the presence of pointing should have little effect on the recognition of high-frequency words but should aid the recognition of low-frequency words. In the study by McCusker et al. (1981), the finding of a stronger wordfrequency effect for visual than for auditory presentation could be interpreted to indicate either that auditory presentation facilitates recognition of low-frequency words or that it hinders the recognition of high-frequency words. In the present study, the amount of phonological information was manipulated visually, thus affording a choice between visual and phonological access to the lexicon. Therefore, if the expected frequency $\times$ pointing interaction is obtained, it will be possible to see whether this is due to the presence of pointing facilitating phonological encoding (and thus aiding processing of low-frequency words) or to the presence of pointing inducing phonological encoding (and thus impairing recognition of highfrequency words).

The second aim pertained to the nature of the phonological recoding process. If the presence of pointing is found to aid the recognition of low-frequency words, examination of the interaction between pointing and word length should clarify the process by which a printed word is coded into a phonological representation. Two hypotheses may be contrasted. According to the first, the "piecemeal hypothesis," phonological recoding is carried out serially from the beginning to the end of the word by the application of grapheme-to-phoneme conversion rules (cf. Coltheart, 1978). McCusker et al. (1979), for example, proposed that high- and low-frequency words differ at the stage at which orthographic features are combined into higher order units en route to lexical access: High-frequency words are encoded and accessed using a holistic, visually based code, whereas low-frequency words rely on a slower, phonologically mediated representation, which is carried out serially, in a left-to-right fashion. The second hypothesis, the "whole-word hypothesis," states that the derivation of a phonological code for a printed word may be carried out all at once, on the basis of its holistic pattern, by accessing the corresponding entry in a vocabulary of stored phonological representations. This hypothesis has been proposed for reading aloud (Glushko, 1979; Kay \& Marcel, 1981; Marcel, 1980), but may be extended to apply to the phonological encoding assumed to mediate recognition of lowfrequency words.

If the piecemeal, serial hypothesis is correct, we should predict that whenever pointing is observed to aid word recognition, this effect should increase with word length. Thus, a triple interaction of frequency $x$ pointing $\times$ length should be expected. The whole-word hypothesis, 
on the other hand, implies that the effect of pointing should be indifferent to word length.

A third aim involved the effects of context. Adults who study Hebrew as a foreign language are often astonished at the ease with which fluent Hebrew readers can read unpointed text. It is quite possible that connected discourse contains sufficient contextual cues that facilitate phonological recoding when this is necessary for comprehension, and helps resolve phonological ambiguities. To examine this idea, target words were preceded by semantically related or semantically unrelated prime words. If semantic context aids the phonological recoding process, we should expect that, for low-frequency words, the presence of a related context should compensate for the absence of pointing.

\section{METHOD}

\section{Stimulus Materials}

For experimental targets, 128 Hebrew words were used, representing two levels of word frequency and four levels of word length. The high-frequency words were selected from the most frequently used 3,000 words with usage frequency of over 8 in 200,000 (Balgur, 1968). The low-frequency words included either words that were listed in Balgur with a smaller frequency of usage or words not listed in this corpus and judged to be infrequently used but well within the vocabulary of the student population. Word length varied from two to five consonantal letters. Thus, the word barzel, for example, is a four-letter word, since it is spelled as brzl.

All words satisfied the following conditions. First, when unpointed, they allowed for only one pronunciation. This means that, for all words, pointing did not resolve any lexical ambiguity. Second, none of the words included the letters $\mathrm{Vav}$ or Yod, which are often used to convey vowel information. Third, none of the words included a silent letter, that is, a letter that is normally unpronounced.

Eight words in each frequency $\times$ length cell were transformed into pronounceable nonwords by replacing one of the letters with another letter (not including Yod or Vav). In their pointed form, the nonwords had the same pointing as the original words from which they were derived.

An additional set of 16 words was compiled for filler targets. Each contained the letters Yod or Vav with a vowel function. These were included to make the list of targets more representative of Hebrew words. Eight of these were transformed into nonwords by changing any one of the letters except the Yod or the Vav.

A semantically related prime word was selected for each of the experimental target words and for half of the filler target words. This was done on the basis of intuitive judgments. An additional set of words was compiled to serve as primes for the nonword targets and as unrelated primes for the rest of the filler word targets.

All stimuli were prepared using Letraset Stam HEB 520 letters. They were then photographed, and negative slides (white on black) were used. All primes were pointed to prevent phonological, and often lexical, ambiguity. Experimental target words appeared in both a pointed and an unpointed form.

\footnotetext{
Apparatus

The stimuli were rear-projected with the aid of two Kodak Carousel slide projectors placed one directly above the other, and slightly tilted so that they projected on the same area of the screen. The first was used to project primes and the second to project targets. Both primes and targets were identically centered on the screen. The subjects sat approximately $170 \mathrm{~cm}$ from the screen so that the 14-mm letter height subtended a visual angle of approximately $0.5^{\circ}$. The luminance of the letters was approximately $37 \mathrm{~cd} / \mathrm{m}^{2}$.
}

\section{Design and Procedure}

The experimental word targets formed a $2 \times 4$ frequency $\times$ length factorial with eight words in each cell. The experimental nonwords were similarly constructed, with frequency referring to frequency of usage of the base words. The eight targets in each of the cells were divided into four sets so that, for each subject, each set apappeared in all four combinations across every group of four subjects.

The target words were presented in exactly the same order for all subjects, but each word was pointed for half of the subjects and unpointed for the other half. For each of the two groups, half of the pointed words and half of the unpointed words were preceded by their related primes, and the rest were preceded by unrelated primes. The unrelated pairs were generated by pairing each of the ,target words with one of the remaining 31 primes of the unrelated set. The list was organized into eight blocks of 18 items each, each block including 2 filler items and 16 experimental items. The latter included 2 items in each of the frequency $\times$ length levels, 1 of them a word and 1 a nonword. The four context $x$ pointing combinations were equally represented in each block for the experimental items. The order of the items was otherwise random.

The experiment was carried out in a dimly lit room. The subjects were told that they had to classify strings of letters as words or nonwords as quickly as they could, and that each string would be preceded by a pointed word that would serve as a warning signal. They were told that half of the target strings were pointed and half were not, and that the pointed and unpointed stimuli were randomly mixed. They were also informed that when a word was pointed, pointing was always correct, and that none of the pointed nonwords would make a word if pointing were changed or eliminated.

The experiment began with 24 practice trials followed by the experimental trial. After the first 72 experimental trials, the subjects were given a short rest, during which the slide trays were switched.

Each prime appeared for $500 \mathrm{msec}$ and was replaced after a 100 msec interval by the target, which remained in sight for $1,000 \mathrm{msec}$. The subjects classified the letter string by pressing one key with the right index finger for words and another key with the left index finger for nonwords. Response time from the onset of the target was measured on an electronic timer to the nearest millisecond. There was a 3,300-msec interval from the offset of the target to the onset of the next trial, during which interval the slides were replaced.

\section{Subjects}

Forty-eight University of Haifa students, 15 males and 33 females, participated in the study. Of these, 35 participated for course credit, and the rest were paid for their effort.

\section{RESULTS}

We shall first examine the results for words and then proceed to those for nonwords. In all the analyses to be reported, only experimental strings were included.

\section{Effects of Pointing and Semantic context}

Figure 1 presents mean precentages of errors and mean latencies for correct responses for low-frequency and high-frequency words as a function of type of context and presence or absence of pointing.

We shall first look at the error data. A frequency $x$ context $X$ pointing analysis of variance was carried out twice, collapsing first over subjects and then over items (Clark, 1973). The F ratios for these analyses are designated $F_{s}$ and $F_{i}$ respectively. The results indicated the following effects. Low-frequency words yielded $13.0 \%$ er- 


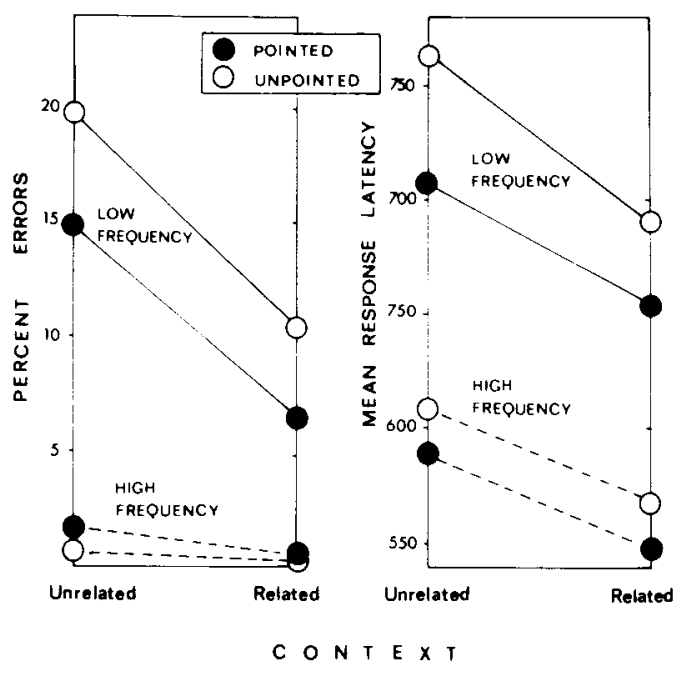

Figure 1. Mean percent errors and response latency (in milliseconds) for word targets as a function of word frequency, presence or absence of pointing, and type of context.

rors, whereas high-frequency words yielded $0.8 \%$ $\left[F_{s}(1,47)=92.51, p<.001\right.$, and $F_{i}(1,62)=34.43$, $p$ $<.001]$. A related context reduced error rate by $4.8 \%$ $\left[\mathrm{F}_{\mathrm{s}}(1,47)=32.89, \mathrm{p}<.001\right.$, and $\mathrm{F}_{\mathrm{i}}(1,62)=27.60, \mathrm{p}$ $<.001]$. Pointing reduced percent errors by $2.0 \%$ on the average $\left[F_{s}(1,47)=4.79, p<.05\right.$, and $F_{i}(1,62)=3.22$, $\mathrm{p}<.10]$. In addition, both the frequency $\times$ context and the frequency $\times$ pointing interactions were significant. The frequency $\times$ context interaction yielded $F_{s}(1,47)=$ $20.23, \mathrm{p}<.001$, and $\mathrm{F}_{\mathrm{i}}(1,62)=18.27, \mathrm{p}<.001$. A related context reduced incidence of errors by $8.7 \%$ for low-frequency words, and by only $0.9 \%$ for highfrequency words, relative to an unrelated context. The frequency $\times$ pointing interaction yielded $F_{s}(1,47)=9.02$, $\mathrm{p}<.005$, and $\mathrm{F}_{\mathrm{i}}(1,62)=5.85, \mathrm{p}<.02$. The presence of pointing reduced incidence of errors by $4.6 \%$ for lowfrequency words and increased incidence of errors by $0.7 \%$ for high-frequency words.

In view of the interactions involving word frequency, separate analyses of variance were carried out for highfrequency and low-frequency words. For high-frequency words, the effect of pointing was not significant in either a subject-based or an item-based analysis, whereas the effect of context was significant only in the subject-based analysis $[F(1,47)=4.81, p<.05]$. For low-frequency words, on the other hand, both analyses yielded significant words for context $\left[F_{s}(1,47)=27.73, p<.001\right.$, and $\left.F_{i}(1,131)=24.90, p<.001\right]$, as well as for pointing $\left[\mathrm{F}_{\mathrm{s}}(1,47)=7.12, \mathrm{p}<.02\right.$, and $\mathrm{F}_{\mathrm{i}}(1,31)=4.61, \mathrm{p}<$ $.01]$. It should be noted that the effects of pointing and context were almost perfectly additive for the lowfrequency words (Figure 1).

Turning now to response latency, a frequency $\times$ pointing $X$ context analysis of variance indicated significant main effects for all three factors. Correct responses to lowfrequency words required $125 \mathrm{msec}$ more than responses to high-frequency words $\left[\mathrm{F}_{\mathrm{s}}(1,47)=481.19, \mathrm{p}<.001\right.$, and $\left.\mathrm{F}_{\mathrm{i}}(1,62)=90.32, \mathrm{p}<.001\right]$. A related context shortened response time by $51 \mathrm{msec}$ on the average $\left[F_{s}(1,47)\right.$ $=113.15, \mathrm{p}<.001$, and $\left.\mathrm{F}_{\mathrm{i}}(1,62)=62.19, \mathrm{p}<.001\right]$. The net benefit due to the presence of pointing amounted to $33 \mathrm{msec}\left[\mathrm{F}_{\mathrm{s}}(1,47)=65.07, \mathrm{p}<.001\right.$, and $\mathrm{F}_{\mathrm{i}}(1,62)$ $=15.40, \mathrm{p}<.001$. The frequency $\times$ context interaction yielded $F_{s}(1,47)=11.16, p<.002$, and $F_{i}(1,62)$ $=5.28, p<.05$. The benefit from a related context was stronger for low-frequency $(63 \mathrm{msec})$ than for highfrequency $(40 \mathrm{msec})$ words. The frequency $\times$ pointing interaction yielded $F_{s}(1,49)=8.85, p<.005$, and $F_{i}(1,62)=2.33$, n.s. The effect of pointing was stronger for low-frequency words $(46 \mathrm{msec})$ than for highfrequency $(20 \mathrm{msec})$ words.

Separate analyses of variance carried out for highfrequency and for low-frequency words indicated the following: For high-frequency words, context yielded $\mathrm{F}_{\mathrm{s}}(1,47)=57.25, \mathrm{p}<.001$, and $\mathrm{F}_{\mathrm{i}}(1,31)=23.19, \mathrm{p}<$ .001 , and pointing yielded $\mathrm{F}_{\mathrm{s}}(1,47)=15.64, \mathrm{p}<.001$, and $F_{\mathrm{i}}(1,31)=4.65, \mathrm{p}<.05$. The context $\times$ pointing interaction was not significant. For the low-frequency words, context yielded $F_{s}(1,47)=92.04, p<.001$, and $\mathrm{F}_{\mathrm{i}}(1,31)=39.08, \mathrm{p}<.001$, and for pointing, $\mathrm{F}_{\mathrm{s}}(1,47)$ $=46.53, \mathrm{p}<.001$, and $\mathrm{F}_{\mathrm{i}}(1,31)=10.74, \mathrm{p}<.01$. Although the presence of pointing produced a $57-\mathrm{msec}$ benefit for unrelated trials and only $35-\mathrm{msec}$ for related trials, the context $\times$ pointing interaction was not significant $\left[F_{s}<1\right.$ and $\left.F_{i}(1,31)=1.47\right]$.

Taken together, the results for both percent errors and response latency indicate the following: First, the presence of pointing appears to aid the processing of low-frequency more than that of high-frequency words. This is consistent with the idea that phonological mediation is involved to a greater extent in the recognition of low-frequency than in the recognition of high-frequency words. Second, although the presence of pointing improves the recognition of low-frequency words, it does not impair the recognition of high-frequency words, suggesting that it aids phonological encoding but does not necessarily induce it. Third, there was no significant pointing $\times$ context interaction to support the hypothesis that semantic context helps resolve phonological ambiguity.

\section{The Effects of Word Length}

Since a sizable pointing effect was observed for lowfrequency words, examination of the pointing $\times$ word length interaction may be instructive. Assuming, with McCusker et al. (1979), that the visually based access of high-frequency words is carried out in whole word units, whereas the phonologically based access of low-frequency words is carried out serially, we should expect a triple interaction of frequency $\times$ pointing $\times$ length.

A frequency $\times$ context $\times$ pointing $\times$ length analysis of variance on percent errors indicated no significant effects for either the pointing $\times$ length or the frequency $x$ pointing $x$ length interactions. Error rate for the highfrequency words revealed few effects of pointing and length. For low-frequency words, the means for lengths $2,3,4$, and 5 , respectively, were $15.1,18.7,20.3$, and 
6.8 for the unpointed spelling, and $13.5,13.0,9.9$ and 6.2 for the pointed spelling. Although for lengths $2-4$ the effect of pointing appears to increase with word length, five-letter words exhibited the smallest advantage for pointed over unpointed spelling, and, as noted, the pointing $\times$ length interaction was not significant.

Figure 2 presents mean response time for correct responses as a function of word frequency, word length, and pointing. A subject-based four-way analysis of variance was carried out on these results. In this analysis, it should be stressed, there were only two observations per cell for each subject, and 17 of the 1,536 subject means were missing because both responses were incorrect. The results indicated significant main effects for frequency, context, and pointing but no significant effect for word length $\left[F_{s}(3,141)=1.29\right]$. Apart from the frequency $x$ context and the frequency $\times$ pointing interactions, which were significant, the only other significant interaction was that between frequency and length $[\mathrm{F}(3,141)=18.03$, $p<.001]$. As may be seen in Figure 2, it appears that, if anything, it is for high-frequency words that response latency tends to increase with increasing word length, whereas for low-frequency words, response time tends to decrease with increasing word length.

In conclusion, examination of the effects of word length does not support either of the following hypotheses: (1) that the effect of word length is stronger for lowfrequency than for high-frequency words, or (2) that for low-frequency words, the benefit from pointing increases with increasing word length. If pointing is assumed to aid the derivation of a phonological code from a printed word, the results seem to suggest that this derivation is carried out all at once, for the entire word as whole.

\section{Analysis of Nonwords}

We shall turn now to the responses to experimental non-

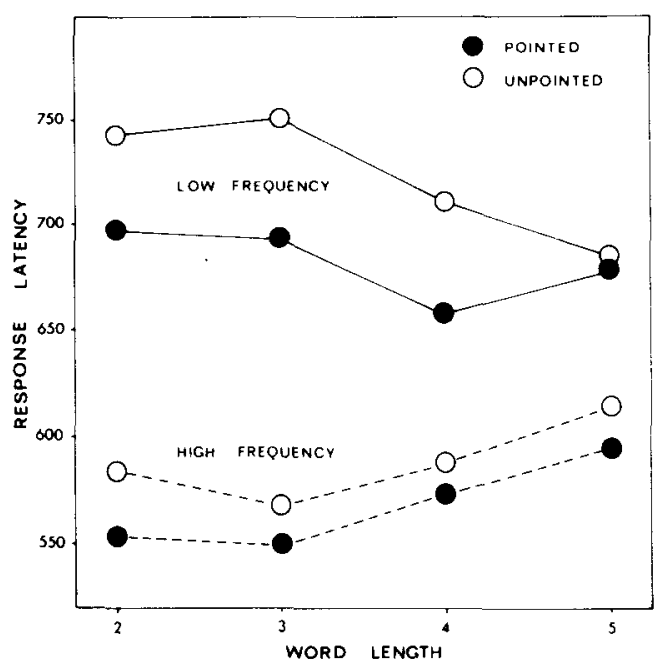

Figure 2. Mean response latency (in milliseconds) for pointed and unpointed high- and low-frequency words as a function of string length. word targets. Three nonwords that were misidentified as words (apparently because the identity of a single letter was mistaken) were eliminated from the analyses. For the rest of the nonwords, Figure 3 presents mean percent errors and mean response latency for correct responses as a function of frequency (of base word), pointing, and string length.

The results seem to indicate a certain degree of speedaccuracy trade-off for the effects of pointing. A frequency $\times$ pointing $\times$ length analysis of variance on percent errors yielded: $F_{s}(1,47)=12.70, p<.001$, and $F_{i}(1,56)$ $=6.24, \mathrm{p}<.05$, for frequency; $\mathrm{F}_{\mathrm{s}}(1,46)=8.03, \mathrm{p}<$ .01 , and $\mathrm{F}_{\mathrm{i}}(1,56)=5.36, \mathrm{p}<.05$, for pointing; and $\mathrm{F}_{\mathrm{s}}(1,141)=14.83, \mathrm{p}<.001$, and $\mathrm{F}_{\mathrm{i}}<1$ for length. None of the interactions was significant. High-frequency nonwords elicited more errors $(9.5 \%)$ than low-frequency nonwords $(6.3 \%)$, and unpointed nonwords elicited more errors $(9.4 \%)$ than pointed nonwords $(6.3 \%)$. The effects of string length were not systematic, with the highest proportion of errors obtained for two-letter nonwords $(12.8 \%)$ and the lowest for four-letter nonwords $(4.3 \%)$.

A similar analysis on response time yielded: $F_{s}(1,47)$ $=3.05$, n.s. , and $F_{i}<1$ for frequency; $F_{s}(1,47)=3.18$, n.s., and $\mathrm{F}_{\mathrm{i}}(1,56)=1.48 ;$ n.s. for pointing; and $\mathrm{F}_{\mathrm{s}}(3,141)$ $=7.46, p<.001$, and $F_{i}(3,56)=1.86$, n.s., for length. None of the interactions was significant. As Figure 3 suggests, the presence of pointing, if anything, tends to slow down responses. The effect of string length appears to be entirely due to the five-letter strings requiring more time to respond to than shorter nonwords. When five-letter nonwords are eliminated from the subject-based analysis, the results indicate $F<1$ for string length.

In sum, as far as the main issues of the present study are concerned, two findings are worth noting. First, although pointing reduced error rate, it did not aid speed of responding to nonwords. This is rather surprising in view of the fact that low-frequency words did seem to benefit from pointing. Second, there was no indication that the effects of pointing interact with those of string length in a manner consistent with the piecemeal phonological recoding hypothesis.

\section{DISCUSSION}

The results will be discussed with reference to four issues: the possibility of greater phonological mediation for low-frequency words, the nature of the phonological encoding process, the effect of semantic context on reading, and the process underlying response to nonwords.

\section{Phonological Mediation and Word Frequency}

The idea that phonological mediation is involved to a greater extent in the recognition of low-frequency than in the recognition of high-frequency words was investigated by comparing the effects of pointing on these two types of words. The assumption was that pointing provides phonological information. This assumption is supported by the finding (Koriat, 1984) that pointing does 


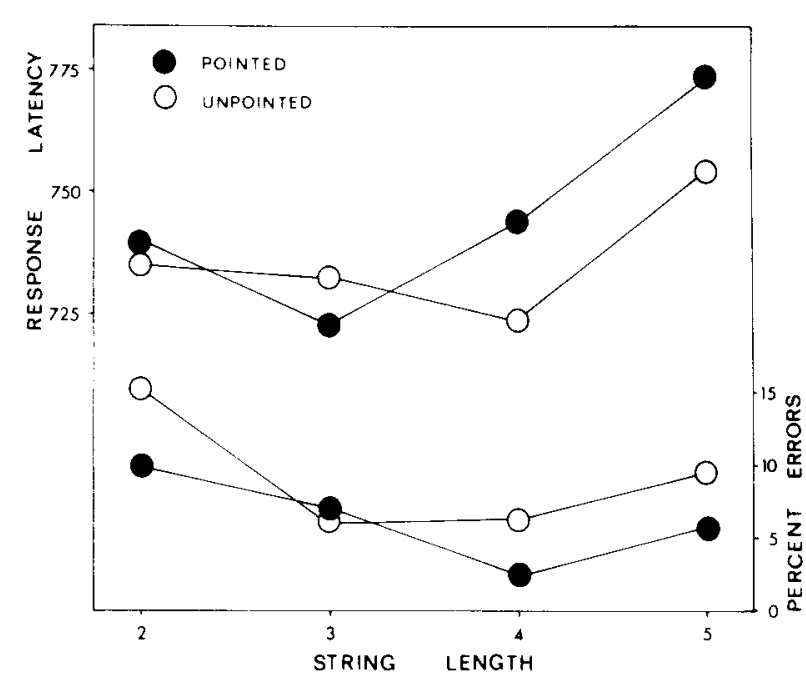

Figure 3. Mean percent errors and response latency (in milliseconds) for pointed and unpointed nonword targets.

not aid lexical decision but does aid speed of pronunciation. Furthermore, a recent study (Koriat, in press) indicated that for laterally presented words pointing had an adverse effect on lexical decision and reading aloud. This result suggests a distinction between the visual and the phonological contributions of pointing: With peripheral vision, pointing seems to tax visual analysis and to impair word recognition, and this impairment offsets whatever phonological benefit might accrue to pointing.

The results for the error and reaction time data of the present study indicated a stronger beneficial effect of pointing for low-frequency than for high-frequency words. Stated differently, the size of the word-frequency effect was larger for unpointed than for pointed words. For the unpointed spelling, low-frequency words elicited $14.7 \%$ more errors than high-frequency words, and correct responses took $139 \mathrm{msec}$ longer. The respective figures for the pointed spelling were $9.5 \%$ and $113 \mathrm{msec}$. This is consistent with the hypothesis that phonological recoding is involved to a greater extent in the recognition of low-frequency words than in the recognition of highfrequency words. It is also compatible with McCusker et al.'s finding (1979) of a smaller word-frequency effect for auditory than for visual presentation. Our results additionally suggest that the reduction in the wordfrequency effect apparently stems from a benefit to lowfrequency words rather than a cost to high-frequency words.

The rate of errors found for the low-frequency unpointed words in the unrelated-prime condition was very high, $19.8 \%$. The observation that this rate drops to $6.5 \%$ when pointing and a related context are added (Figure 1) suggests that the high rate of errors is not due to the lack of familiarity with the words employed. According to the assumptions underlying the present study, these errors reflect, in part, incorrect phonological encodings that result in "nonword" decisions. Indeed, in an exploratory study, subjects who were asked to pronounce the strings quickly but correctly evidenced $15 \%-20 \%$ mispronunciations for the low-frequency unpointed words. On the other hand, when the nonwords were eliminated and subjects were urged to read the words correctly, rate of mispronunciations was negligible. This pattern of results suggests that in the lexical decision task the instructions to respond quickly together with the presence of nonwords in the list resulted in the tendency to respond NO to low-frequency unpointed words when these were initially assigned incorrect phonologies.

The observation that, even for high-frequency words, pointing had a beneficial effect on response time is inconsistent with the previous results of no pointing effects for words of comparable frequencies (Koriat, 1984). Although the effect was small (a 20 -msec advantage), it was quite reliable across subjects, with 37 of the 48 subjects showing an overall advantage for the pointed spelling. It is possible that the inclusion of low-frequency words in the present study induced subjects to adopt a phonological strategy, and this may have been detrimental to high-frequency words. Consistent with this interpretation (see McCusker et al., 1981) is the finding of Glanzer and Ehrenreich (1979) that high-frequency words are processed faster when they appear alone than when they are mixed with low-frequency words.

\section{The Phonological Encoding Process}

The results yielded little support for the hypothesis that when pointing has a beneficial effect (e.g., low-frequency words), the size of this effect increases with increasing word length. If the presence of pointing is assumed to aid in the derivation of a phonological code, then it would seem that this derivation is carried out all at once on the basis of the word as a whole.

This idea is consistent with recent work on reading aloud (e.g., Glushko, 1979; Kay \& Marcel, 1981; Marcel, 1980) that suggests that the phonological representation of a word may be obtained by accessing its corresponding entry in a phonological lexicon containing the stored pronunciations of all known words. Furthermore, studies with patients with severe semantic loss suggest that phonological reading does not rest exclusively on the application of grapheme-to-phoneme conversion rules. Thus, a patient studied by Schwartz, Saffran, and Marin (1980) could read aloud irregular words that she could not comprehend. Data by Shallice, Warrington, and McCarthy (1983) suggest that reading without semantics may use orthographic units that are larger than the grapheme. Coltheart, Masterson, Byng, Prior, and Riddoch (1983) observed homophone confusions with irregularly spelled words (e.g., bury - "a fruit on a tree"). These were taken to suggest a form of phonological reading that uses direct access from visual input logogens to output logogens without grapheme-to-phoneme conversion.

In Marcel's (1980) formulation, access to the phonological lexicon is seen as a precursor to pronunciation, and this lexicon is conceived of as an output lexicon (see 
Marcel 1980, Figure 11.2). In the present formulation, in contrast, the phonological lexicon is conceived of as an input lexicon that may mediate access to meaning. $\mathrm{Ob}$ viously, it is important to determine whether one and the same phonological lexicon mediates access to both meaning and pronunciation (see Allport, 1984, and Allport \& Funnell, 1981). If it does, it would mean that when access to the internal lexicon of meanings is phonologically based (as with low-frequency words), the retrieval of a word's meaning and the retrieval of its pronunciation share a common route. Two interesting predictions follow from this assumption. First, if the recognition of low-frequency words requires access to the same phonological entry that underlies pronunciation, whereas the recognition of highfrequency words does not, then the advantage of highfrequency over low-frequency words should be smaller for pronunciation than for lexical decision latencies. This has indeed been found to be the case (e.g., Frederiksen \& Kroll, 1976; Richardson, 1976). Second, in the previous study (Koriat, 1984), pointing aided pronunciation but not lexical decision for high-frequency words, whereas in the present study, pointing aided lexical decision, particularly for low-frequency words. If the effects of pointing in the two cases involves access to the same phonological lexicon, we should expect the frequency $x$ pointing interaction obtained in the present study to decrease or even disappear when a pronunciation task is employed.

\section{Semantic Context and Phonological Encoding}

It was hypothesized that the presence of a related semantic context aids phonological encoding and thus compensates for the absence of pointing. This hypothesis was not supported by the data. The observation that the effects of pointing and context are largely additive even for lowfrequency words is not easy to interpret. Assume that pointing aids access to the word's corresponding entry in the phonological lexicon. Assume further that the effect of a related context is to preactivate a lexical entry corresponding to the word target. The additivity of the two effects may then imply that this latter entry is different from the phonological entry and probably represents an address in a more abstract lexicon of meanings. This would imply that a preactivation of an entry in the semantic lexicon does not spread out to the corresponding entry in the phonological lexicon. This implication is worthy of further examination. Kay and Marcel (1981), for example, found that the pronunciation of a phonologically ambiguous nonword (such as NOUCH) was biased by a word that preceded it that had the same ambiguous segment (e.g. COUCH or TOUCH). It would be of interest to see whether the pronunciation of the nonword NOUCH is affected by the word SOFA in the same way that it is affected by the word COUCH.

\section{Response to Nonwords}

The observation that pointing affects response time to words but at the same time does not affect response time to nonwords is rather surprising. This finding is inconsistent with the proposal that nonwords are pronounced by the application of grapheme-to-phoneme conversion rules, and that NO responses in a lexical decision task evidence the operation of phonological recoding more than YES responses (cf. Coltheart, 1978). If phonological recoding is achieved through the application of print-tosound conversion rules, then unpointed nonwords should prove quite difficult to categorize, since they permit many phonological representations, all of which must be tested before a NO decision can be safely reached. Pointing should therefore have a beneficial effect, since it reduces greatly the number of phonological representations to be tested.

Consider, on the other hand, a model that posits a whole-word access to the phonological lexicon. We may assume that when a letter string is presented, an attempt is made to locate its corresponding entry in either the visual or the phonological lexicon. When a word is of low frequency and cannot be recognized visually, presence of pointing may aid in locating its corresponding entry in the phonological lexicon. If no entry is found after a predetermined deadline, a NO response is issued (cf. Coltheart, Davelaar, Jonasson, \& Besner, 1977). This deadline must, of course, be set at a value that is greater than the time required for accessing the phonological code for a low-frequency unpointed word. (Indeed, correct response to nonwords averaged $745 \mathrm{msec}$, compared with $727 \mathrm{msec}$ for unpointed low-frequency words.) This process should result in pointing effects for YES responses but not for NO responses.

\section{CONCLUSION}

In conclusion, the results of the present study are consistent with the idea that word frequency may affect choice of mediating process for lexical access. They suggest that phonological mediation tends to be involved to a larger extent in the processing of low-frequency than in the processing of high-frequency words. The phonologically mediated process appears to involve, first, retrieving the appropriate representation of the printed word from the phonological lexicon, and then using this representation to gain access to the word's entry in the semantic lexicon. Failure to locate an entry in the phonological lexicon results in a nonword decision. Priming of a word's entry in the semantic lexicon does not appear to aid access to the word's entry in the phonological lexicon.

Although the present study provided some information regarding the process underlying the recognition of lowfrequency words, several major questions still remain. The first concerns the effect of pointing. If the derivation of a phonological code in Hebrew were carried out on the basis of grapheme-to-phoneme conversion rules, then the advantage of pointed over unpointed spelling could be readily explained. However, since, as I proposed, this derivation appears to be achieved by looking the word up in a phonological lexicon, how pointing may aid in 
such a process is not entirely clear. One possibility, which is currently being explored, is that the effect of pointing is to inhibit phonological analogies that would compete with the correct phonological encoding of a word. If this is correct, it would be possible to specify the exact conditions under which pointing may aid lexical access and pronunciation.

The second issue concerns the effects of word frequency. Why phonological mediation should be involved to a greater extent in the recognition of low-frequency than in the recognition of high-frequency words is not at all clear. Marcel (1980), for example, proposed that, in beginning readers, the lower the frequency of a word, the less likely it is to acquire an orthographic address. Similarly, McCusker et al. (1981, p. 223) implied that lowfrequency words do not have visual representations, and therefore cannot be accessed directly. However, why a low-frequency word should be more likely to have a phonological representation than a visual representation is not clear. Future research must try to explain the interaction between word frequency and mode of lexical access.

A third issue concerns the role that the phonological lexicon plays in word recognition and pronunciation. The recent work on word pronunciation (Glushko, 1979; Marcel, 1980) has contributed valuable knowledge regarding the process by which phonological representations are obtained. If the results on reading aloud are found to have implications regarding lexical access, these implications must be incorporated into theories of word recognition.

Finally, I should stress that whether the conclusions reached in the present work are specific to Hebrew reading and Hebrew readers or are generalizable to other languages as well is still unclear. If they are found to have some generality, Hebrew might prove valuable for the study of some of the basic issues in reading.

\section{REFERENCES}

AllPoRT, D. A. (1984). Speech production and comprehension: One lexicon or two. In W. Prinz \& A. F. Sanders (Eds.), Cognition and motor processes. Berlin: Springer-Verlag.

Allport, D. A., \& FunNell, E. (1981). Components of the mental lexicon. Philosophical Transactions of the Royal Society, 295, 397410.

Baron, J. (1973). Phonemic stage not necessary for reading. Quarterly Journal of Experimental Psychology, 25, 241-246.

BALGUR, R. (1968). [The basic word list for elementary schools]. Ramat Gan, Israel: Otsar Hamoreh.

Bradshaw, J. L. (1975). Three interrelated problems in reading: A review. Memory \& Cognition, 3, 123-134.

CLARK, H. H. (1973). The language-as-fixed-effect fallacy: A critique of language statistics in psychological research. Journal of Verbal Learning and Verbal Behavior, 12, 335-359.
Coltheart, M. (1978). Lexical access in simple reading tasks. In G. Underwood (Ed.), Strategies of information processing. London: Academic Press.

Coltheart, M., Davelaar, E., Jonasson, J. T., \& Besner, D. (1977). Access to the internal lexicon. In S. Dornic (Ed.), Attention and performance $V I$. Hillsdale, NJ: Erlbaum.

Coltheart, M., Masterson, J., Byng, S., Prior, M., \& Riddoch, J. (1983). Surface dyslexia. Quarterly Journal of Experimental Psychology, 35A, 469-495.

FREDERIKSEN, J., \& KROLL, J. F. (1976). Spelling and sound: Approaches to the internal lexicon. Journal of Experimental Psychology: Human Perception and Performance, 2, 361-379.

GLANZER, M., \& EHRENREICH, S. L. (1979). Joumal of Verbal Learning and Verbal Behavior, 18, 381-398.

GLushKo, R. J. (1979). The organization and activation of orthographic knowledge in reading aloud. Journal of Experimental Psychology: Human Perception and Performance, 5, 674-691.

Hardyck, C. D., \& Petrinovich, L. F. (1970). Subvocal speech and comprehension level as a function of the difficulty level of the reading material. Journal of Verbal Learning and Verbal Behavior, 9, 647-652.

KAY, J., \& MARCEL, A. (1981). One process, not two, in reading aloud: Lexical analogies do the work of non-lexical rules. Quarterly Journal of Experimental Psychology, 33, 397-413.

Kleiman, G. M. (1975). Speech recoding in reading. Journal of Verbal Learning and Verbal Behavior, 14, 323-339.

KoRIAT, A. (1984). Reading without vowels: Lexical access in Hebrew. In H. Bouma \& D. G. Bouwhuis (Eds.), Attention and performance $X$ : Control of language processes (pp. 227-242). Hillsdale, NJ: Erlbaum.

Koriat, A. (in press). Lateralization effects in reading pointed and unpointed Hebrew. British Journal of Psychology.

MARCEL, A. (1980). Surface dyslexia and beginning reading: A revised hypothesis of the print and its impairments. In M. Coltheart, K. Patterson, \& J. C. Marshall (Eds.), Deep dyslexia. London: Routledge \& Kegan Paul.

McCusker, L. X., Holley-WilcoX, P., \& Hillinger, M. L. (1979, April). Frequency effects in auditory and visual word recognition. Paper presented at the meeting of the Southwestern Psychological Association, San Antonio, TX.

McCusker, L. X., Hillinger, M. L., \& Bias, R. G. (1981). Phonological recoding and reading. Psychological Bulletin, 89, 217-245.

Meyer, D. E., SchvaneveldT, R. W., \& Ruddy, M. G. (1974). Functions of graphemic and phonemic codes in visual word recognition. Memory \& Cognition, 2, 309-321.

Navon, D., \& Shimron, J. (1981). Does word naming involve grapheme to phoneme translation? Evidence from Hebrew. Journal of Verbal Learning and Verbal Behavior, 20, 97-109.

RicharDson, J. T. E. (1976). The effects of stimulus attributes upon latency of word recognition. British Journal of Psychology, 67, 315-325.

Rubenstein, H., Lewis, S. S., \& Rubenstein, M. A. (1971). Evidence for phonemic recoding in visual word recognition. Journal of Verbal Learning and Verbal Behavior, 10, 645-657.

SchwarTZ, M. F., Saffran, E. M., \& Marin, O. S. M. (1980). Fractionating the reading process in dementia: Evidence for word-specific print-to-sound associations. In M. Coltheart, K. Patterson, \& J. C. Marshall (Eds.), Deep dyslexia. London: Routledge \& Kegan Paul.

Shallice, T., Warrington, E. K., \& McCarthy, R. (1983). Reading without semantics. Quarterly Journal of Experimental Psychology, 35A, 111-138.

(Manuscript received June 8, 1984; revision accepted for publication July 10, 1984.) 